\title{
Catarata e diabetes mellitus tipo 1
}

\author{
Cataract and type 1 diabetes mellitus
}

\author{
Melissa Manfroi Dal Pizzol ${ }^{1}$ \\ Jorge Freita Esteves ${ }^{2}$ \\ Caio Augusto Sccoco ${ }^{3}$ \\ Murilo Felix Roggia ${ }^{4}$ \\ Carolina Maurente da Rosa ${ }^{5}$ \\ José Humberto Franco Lambert ${ }^{6}$ \\ Luís Henrique Canani ${ }^{7}$
}

\section{RESUMO}

Objetivo: Avaliar a prevalência de catarata e seus fatores de risco em uma população portadora de diabetes mellitus tipo 1 (DM1). Métodos: Estudo de casos e controles de um banco de dados de 181 pacientes (362 olhos) com diagnóstico de diabetes mellitus tipo 1 . Os pacientes foram classificados como casos quando apresentavam diagnóstico de catarata. As variáveis estudadas foram a presença ou não de retinopatia diabética, tratamento com panfotocoagulação, presença de hipertensão arterial sistêmica e neuropatia periférica, colesterol total, triglicerídios séricos, HDL, LDL, índice de massa corporal, creatinina sérica, albuminúria, hemoglobina glicosilada e glicemia de jejum. Resultados: A prevalência de catarata foi de 19,9\%. Na análise univariada foi encontrada associação estatisticamente significativa $(\mathrm{p}<0,005)$ entre a presença de catarata e retinopatia diabética, neuropatia periférica, creatinina sérica elevada, panfotocoagulação e hipertensão arterial. Após a análise de regressão logística a variável que permaneceu com associação estatisticamente significativa foi a presença de retinopatia diabética. Conclusão: A prevalência de catarata foi de $19,9 \%$, sendo a presença e a gravidade da retinopatia diabética os principais fatores de risco para desenvolvimento desta.

Descritores: Catarata/epidemiologia; Retinopatia diabética/complicação; Diabetes mellitus tipo 1; Hipertensão; Hemoglobina A glicosilada; Catarata/etiologia

\section{INTRODUÇ̃̃̃O}

O diabetes é um fator de risco conhecido para o desenvolvimento de catarata. A maior freqüência de opacidade do cristalino em pacientes diabéticos já foi descrita por alguns autores ${ }^{(1-3)}$, sendo a hiperglicemia considerada responsável por esta maior prevalência devido a alterações metabólicas que modificam as proteínas cristalinianas ${ }^{(4-5)}$.

Há poucos estudos na literatura que demonstrem os fatores associados ao surgimento de catarata em diabéticos do tipo 1. No entanto, estudos epidemiológicos já mostraram existir alguns fatores contribuindo para o surgimento de catarata em pacientes diabéticos em geral, dentre os quais pode-se destacar o diabetes tipo $1^{(6-7)}$, o tempo de duração da doença ${ }^{(8-10)}$, a idade avançada no momento do diagnóstico ${ }^{(10-11)}$, a retinopatia avança$\mathrm{da}^{(8,12)}$, o uso de diuréticos ${ }^{(6,12)}$ e o controle metabólico inadequado ${ }^{(8,12-13)}$.

A relação entre o diabetes tipo 2 e o surgimento de catarata pode ser mais difícil de se elucidar uma vez que estes pacientes podem apresentar opacidade cristaliniana relacionada à idade além do fato de que o passado glicêmico e a duração da doença geralmente podem não ser bem documentados. Ao contrário, pacientes com diabetes tipo 1 geralmente apresentam o tempo de duração da doença e o controle metabólico bem determinados ${ }^{(13)}$. Este estudo tem como objetivo avaliar a prevalência de catarata e seus fatores de risco em uma população portadora de diabetes mellitus tipol. 


\section{MÉTODOS}

Estudo de casos e controles de um banco de dados de 181 pacientes (362 olhos) com diagnóstico de diabetes mellitus tipo 1 (DM1) acompanhados no Serviço de Oftalmologia do Hospital de Clínicas de Porto Alegre (HCPA), no período de janeiro de 2004 a março de 2006 através de exame físico, oftalmológico e laboratoriais.

Os pacientes foram estratificados e classificados como casos quando apresentavam diagnóstico de catarata segundo os critérios LOCSII (Lens Opacities Classification System II), enquanto os sem este diagnóstico foram classificados como controles.

As variáveis estudadas foram a presença ou não de retinopatia diabética (RPD), tratamento com panfotocoagulação (PFC), presença de hipertensão arterial sistêmica (HAS) e neuropatia periférica (NPP), colesterol total, triglicerídios séricos, HDL, LDL, índice de massa corporal (IMC), creatinina sérica, albuminúria, hemoglobina glicosilada (HbA1c) e glicemia de jejum. Todas as variáveis foram analisadas no momento do exame oftalmológico considerado no estudo.

Com relação à RPD, os pacientes foram divididos em 3 grupos: grupo 1, pacientes sem RPD; grupo 2, pacientes que apresentavam RPD não proliferativa leve ou moderada; grupo 3, pacientes que apresentavam RPD não proliferativa grave ou proliferativa.

Pacientes tratados com PFC mesmo que incompleta foram analisados no estudo como tendo a variável presente.

A avaliação da neuropatia periférica foi realizada através da verificação dos reflexos profundos do tendão aquileu; sensibilidade vibratória do hálux e índex; sensibilidade fina verificada através do monofilamento (10 gramas) no hálux e índex; sensibilidade grosseira verificada através da distinção da ponta e cabo de uma agulha de insulina no hálux e índex; capacidade de caminhar nos calcanhares. Cada critério ausente foi considerado um ponto, sendo cada membro avaliado isoladamente. A presença de NPP foi considerada quando o somatório dos sinais do exame físico foram maiores que três pontos tanto à direita quanto à esquerda ou apenas dificuldade de deambular nos calcanhares.

Os níveis de pressão sangüínea (PA) utilizados foram as médias da primeira e segunda medidas no exame clínico. HAS foi definida como PA sistólica $\geq 130 \mathrm{mmHg}$ e/ou PA diastólica $\geq 80 \mathrm{mmHg}$ ou qualquer PA na presença de uso de drogas anti-hipertensivas.

Dislipidemia foi considerada quando colesterol total $\geq 200 \mathrm{mg} / \mathrm{dl}$ e/ou LDL $\geq 160 \mathrm{mg} / \mathrm{dl}$ e/ou HDL $\leq 45 \mathrm{mg} / \mathrm{dl} \mathrm{e} /$ ou triglicerídios $\geq 150 \mathrm{mg} / \mathrm{dl}$. Glicemias de jejum $\geq 130 \mathrm{mg} / \mathrm{dl} \mathrm{e} /$ ou HbA1c $\geq 7,0 \%$ foram consideradas elevadas, de acordo com a Associação Americana de Diabetes. O método laboratorial utilizado foi ensaio enzimático colorimétrico.

Albuminúria foi analisada em amostra simples de urina, sendo valores menores que $17 \mathrm{mg} / \mathrm{l}$ considerados anormais, entre 17-174 mg/l microalbuminúria e $\geq 174$ mg/l macroalbuminúria.

Foram excluídos pacientes com mais de 40 anos de idade e com história de trauma ocular ou uveíte.
A análise estatística foi feita com o programa SPSS 12.0. A associação de catarata com RPD, PFC, NPP foi avaliada pelo teste Qui-quadrado. Teste $t$ de Student foi utilizado para as amostras independentes (IMC, colesterol total, HDL, LDL). O teste Mann-Whitney foi utilizado para avaliar as variáveis de distribuição assimétrica (glicemia, HbA1c, triglicerídios, creatinina, albuminúria). Após a análise univariada, foi realizada uma análise de regressão logística com a variável dependente (catarata) e RPD, PFC, HAS, NPP e HbA1c.

\section{RESULTADOS}

Do total de 362 olhos, 72 olhos (19,9\%) apresentaram diagnóstico de catarata, sendo que 290 olhos $(80,1 \%)$ não apresentaram tal diagnóstico. A tabela 1 demonstra as características dos grupos, sendo que apenas a acuidade visual (AV) apresentou diferença estatisticamente significativa entre eles $(\mathrm{p}<0,001)$.

$\mathrm{Na}$ análise univariada foi encontrada associação estatisticamente significativa $(\mathrm{p}<0,005)$ entre a presença de catarata e RPD, neuropatia periférica (NPP), PFC e HAS (Tabela 2). O valor de creatinina também teve diferença estatisticamente significativa entre os grupos, sendo que o valor médio de creatinina no grupo com catarata de $3,5 \mathrm{mg} / \mathrm{dl}$ e no grupo sem catarata de $1,3 \mathrm{mg} / \mathrm{dl}(\mathrm{p}<0,001)$.

As demais variáveis estudadas como glicemia de jejum, HbA1c, IMC, colesterol total, triglicerídios, HDL, LDL, albuminúria não tiveram associação estatística positiva com a presença de catarata (Tabela 3 ).

\begin{tabular}{|lcc|}
\hline \multicolumn{3}{|c|}{ Tabela 1. Características gerais entre os grupos de pacientes com } \\
& e sem catarata & \\
& Grupo & Grupo \\
& com catarata & sem catarata \\
Idade média (anos) & $29,5 \pm 10,4$ & $27,6 \pm 10,3$ \\
Sexo feminino (\%) & $55,5 \%$ & $45,2 \%$ \\
Raça branca (\%) & $94,4 \%$ & $86,2 \%$ \\
Fumo (\%) & $22,2 \%$ & $19,4 \%$ \\
AV média (logMAR) & $0,5 \pm 0,8$ & $0,2 \pm 0,5$ \\
\hline
\end{tabular}

\begin{tabular}{|ccc|}
\hline \multicolumn{3}{|c|}{$\begin{array}{c}\text { Tabela 2. Prevalência de catarata entre as variáveis com associação } \\
\text { estatisticamente significativa } \\
\text { Prevalência de catarata }\end{array}$} \\
RD (\%) & $14,6 \%$ & $\mathrm{p}<0,001$ \\
Grupo 1 & $33,3 \%$ & \\
Grupo 2 e & $36,8 \%$ & $\mathrm{p}<0,017$ \\
NPP (\%) & $18,7 \%$ & \\
Com & & $\mathrm{p}<0,001$ \\
Sem & $33,7 \%$ & \\
PFC (\%) & $15,2 \%$ & $\mathrm{p}<0,004$ \\
Com & & \\
Sem & $29,7 \%$ & \\
HAS (\%) & $15,7 \%$ & \\
Sem & & \\
\multicolumn{2}{|c|}{} \\
\hline
\end{tabular}




\begin{tabular}{|lccc|}
\hline & Tabela 3. Valores médios das variáveis não associadas com catarata \\
& Grupo com catarata & Grupo sem catarata \\
Média de glicemia jejum $(\mathrm{mg} / \mathrm{dl})$ & $192,86 \pm 179,50$ & $177,58 \pm 103,73$ & $9,30 \pm 6,95$ \\
Média de HbA1c $(\%)$ & $8,57 \pm 1,84$ & $174,86 \pm 45,15$ & $\mathrm{p}=0,303$ \\
Média de colesterol total $(\mathrm{mg} / \mathrm{dl})$ & $175,41 \pm 39,70$ & $54,84 \pm 15,96$ & $\mathrm{p}=0,659$ \\
Média HDL $(\mathrm{mg} / \mathrm{dl})$ & $57,00 \pm 18,12$ & $101,79 \pm 36,21$ & $\mathrm{p}=0,925$ \\
Média LDL $(\mathrm{mg} / \mathrm{dl})$ & $98,66 \pm 42,83$ & $99,09 \pm 61,85$ & $\mathrm{p}=0,323$ \\
Média de triglicerídios $(\mathrm{mg} / \mathrm{dl})$ & $96,31 \pm 45,34$ & $24,36 \pm 4,05$ & $\mathrm{p}=0,597$ \\
Média de IMC & $24,32 \pm 3,35$ & $198,16 \pm 63,31$ & $\mathrm{p}=0,947$ \\
Média de albuminúria $(\mathrm{mg} / \mathrm{l})$ & $193,59 \pm 68,54$ & & $\mathrm{p}=0,106$ \\
\hline
\end{tabular}

A prevalência de catarata na população sem RPD (grupo 1) foi de $5,7 \%$, sendo que o grupo 2 apresentou prevalência de $29,9 \%$ e o grupo 3 de $34,7 \%$. A presença de RPD teve associação estatisticamente significativa com o surgimento de catarata, sendo que quanto mais grave fosse a RPD (grupo 3) maior foi a associação com catarata $(\mathrm{p}<0,001)$.

Após a análise de regressão logística a variável independente que permaneceu com associação estatisticamente significativa foi a presença de RPD (grupo 2 e 3), sendo que os pacientes com RPD apresentaram 2,18 vezes mais chance de ter catarata que o grupo sem RPD (grupo 1). Houve uma tendência para a associação de catarata com HAS e NPP (Tabela 4).

\section{DISCUSS ÃO}

Neste estudo encontramos uma prevalência de catarata em diabéticos tipo 1 de $19,9 \%$. O principal fator associado a essa complicação foi a presença de RPD, sendo esta relação tão importante que, quanto mais avançada a retinopatia, maior a associação. Em 1995, alguns autores, avaliando a população do estudo "Wisconsin Epidemiologic Study of Diabetic Retinophathy" (WESDR), observaram uma incidência geral cumulativa em 10 anos de $8,5 \%$ de cirurgia de catarata em pacientes com início do diabetes antes dos 30 anos de idade. Essa incidência aumentava proporcionalmente com a idade dos pacientes, chegando até $27 \%$ quando a idade era igual ou maior do que $45 \operatorname{anos}^{(9)}$. Nesse mesmo estudo foi encontrada uma relação direta do grau de RPD com a formação de catarata, sendo o risco relativo de 3,85 para retinopatia leve, 12,11 para retinopatia moderada e 33,94 para retinopatia proliferativa. Outros autores também demonstram associação positiva entre a presença de RPD e essa complicação, corroborando com os nossos achados ${ }^{(13)}$.

A prevalência de catarata em pacientes pediátricos parece ser mais baixa. Um estudo descreve uma prevalência de 1\% dessa complicação em uma população de 600 pacientes pediátricos com DM1. Nesse trabalho, apesar da ausência de análise estatística, a catarata foi atribuída a distúrbios metabólicos como cetoacidose, hiperosmolaridade e hiperglicemia ${ }^{(14)}$.

A presença de PFC apresentou associação positiva com catarata na análise univariada, sendo possivelmente explicado pelo fato da PFC ser indicada em todos os casos de RPD não

\begin{tabular}{|c|c|c|c|}
\hline & p & Odds ratio & IC $95 \%$ \\
\hline RD (grupo 2 e 3) & 0,010 & 2,18 & $1,20-3,97$ \\
\hline HAS & 0,106 & 1,66 & $0,89-3,08$ \\
\hline NPP & 0,091 & 1,95 & $0,89-4,23$ \\
\hline $\mathrm{Hb} 1 \mathrm{Ac}$ & 0,396 & 0,96 & $0,86-1,06$ \\
\hline
\end{tabular}

proliferativa grave ou RPD proliferativa no ambulatório de DM1 de nosso serviço, no entanto após regressão logística não houve tal associação reafirmando os achados da literatu$\mathrm{ra}^{(9)}$. Uma relação causal entre PFC e catarata não pode ser inferida nesta análise, necessitando para tal um estudo com delineamento específico.

Embora encontrada associação positiva entre HAS e catarata na análise univariada $(p<0,004)$, essa relação foi perdida após a análise multivariada, apesar da tendência à significância. O WESDR, após 14 anos de acompanhamento de pacientes com DM1, demonstrou que os níveis tensionais correlacionam-se com o surgimento e progressão da $\mathrm{RPD}^{(12)}$. Outro estudo descreve uma associação positiva entre catarata e aumento da pressão sistólica em pacientes com DM1, mas não evidencia essa correlação com os níveis de pressão diastóli$\mathrm{ca}^{(9)}$. No entanto, os mesmos autores postulam que o uso de determinadas medicações anti-hipertensivas possam estar implicados na gênese da catarata. Drogas antiadrenérgicas de ação central teriam risco relativo de 5,65 (IC: 2,54-12,50) e diuréticos tiazídicos risco relativo de 2,23 (IC: 1,12-4,44) de causar essa complicação. Sendo assim, não podemos inferir pelo nosso estudo se a HAS seria um fator causal direto ou se estaria relacionado a outros fatores.

A NPP teve associação com catarata na análise univarida $(p<0,01)$ permanecendo com tendência a significância após regressão logística. Não encontramos referência na literatura da relação entre NPP e catarata no DM1. No entanto, postulamos que a presença de NPP implique na presença de uma doença sistêmica mais avançada com um maior acometimento da microcirculação.

Alguns autores analisaram prospectivamente a influência do controle glicêmico em pacientes com DM1 inicialmente sem catarata através do exame de biomicroscopia utilizando densitômetro e encontraram que o principal fator correlacionado 
com o desenvolvimento dessa complicação foi o valor da $\mathrm{HbA1} \mathrm{c}^{(13,15)}$. Em nosso estudo a HbA1c e outras variáveis reconhecidas pela literatura como fatores de risco para catarata em pacientes com DM1 como a glicemia de jejum ${ }^{(9,13,15)}$ e a albuminúria ${ }^{(9)}$ não apresentaram associação com a presença da catarata. Atribuímos a esse achado a possibilidade de nossa análise ser um estudo transversal e retrospectivo.

\section{CONCLUSÃO}

Baseado nos resultados encontrados em nosso estudo, concluímos que a prevalência de catarata foi de $19,9 \%$, sendo a presença e a gravidade da RPD os principais fatores de risco para desenvolvimento desta complicação na população estudada de diabéticos tipo 1. Em virtude dos escassos trabalhos relacionando catarata e DM1, sugerimos a necessidade de estudos prospectivos onde essa complicação seja o desfecho primário, visando interferir nos fatores de risco e diminuir sua incidência.

\section{ABSTRACT}

Purpose: To evaluate the prevalence of cataract and associated risk factors in a diabetic type 1 population. Methods: 181 patients (362 eyes) were evaluated in a case-control study. Cases were classified when cataract was present at the time of the examination. The studied outcomes were the presence of diabetic retinopathy, retinal panphotocoagulation, high blood pressure, peripheral neuropathy, total cholesterol levels, triglyceride levels, HDL, LDL, body mass index, creatinine levels, albuminuria, glycosylated hemoglobin and glycemia levels. Results: Cataract was present in $19.9 \%$ of the studied patients. The association of the presence of cataract and diabetic retinopathy, peripheral neuropathy, elevated levels of creatinine, panphotocoagulation and high blood pressure were statistically significant (univariate analysis). After the logistic regression analysis the presence of cataract was significantly associated with the presence of diabetic retinopathy. Conclusion: The prevalence of cataract was $19.9 \%$ and the presence and severity of the diabetic retinopathy were the main risk factors for its development.

Keywords: Cataract/epidemiology; Diabetic retinopathy/complications; Diabetes mellitus, type 1; Hypertension; Hemoglobin A, glycosylated; Cataract/etiology

\section{REFERÊNCIAS}

1. Kahn HA, Leibowitz HM, Ganley JP, Kini MM, Colton T, Nickerson RS, et al. The Framingham Eye Study. I. Outline and major prevalence findings. Am J Epidemiol. 1977;106(1):17-32.

2. Nielsen NV, Vinding T. The prevalence of cataract in insulin-dependent and non insulin-dependent diabetes mellitus. Acta Ophthalmol (Copenh). 1984; 62(4):595-602.

3. Leske MC, Chylack LT, Wu Sy. The lens opacities case-control study: Risk factors for cataracts. Arch Ophthalmol. 1991;109(2):244-51. Comment in: Acta Ophthalmol. 1991;109(12):1635-6; Arch Ophthalmol. 1991;109(12):1636.

4. Van Boekel MA, Hoenders HJ. Glycation of cristallins in lenses from aging and diabetic individuals. FEBS Lett. 1992;314(1):1-4.

5. Swamy MS, Abraham EC. Glycation of lens membrane intrinsic proteins. Curr Eye Res. 1992;11(9):833-42.

6. Skalka HW, Prchal JT. The effect of diabetes mellitus and diabetic therapy on cataract formation. Ophthalmology. 1981;88(2):117-25.

7. Klein BEK, Klein R, Moss SE. Prevalence of cataracts in a population-based study of persons with diabetes mellitus. Ophthalmology. 1985;92(9):1191-6.

8. Klein BE, Klein R, Lee KE. Diabetes, cardiovascular disease, selected cardiovascular disease risk factors and the 5-year incidence of age-related cataract and progression of lens opacities: The Beaver Dam Eye Study. Am J Ophthalmol. 1998;126(6):782-90.

9. Klein BEK, Klein R, Moss SE. Incidence of cataract surgery in the Wisconsin epidemiologic study of diabetic retinopathy. Am J Ophthalmol. 1995;119(3): 295-300.

10. West SK, Valmadrid CT. Epidemiology of risk factors for age-related cataract. Surv Ophthalmol. 1995;39(4):323-34.

11. Bron AJ, Sparrow J, Brown NA, Harding JJ, Blakytny R. The lens in diabetes. Eye. 1993;7(Pt 2):260-75.

12. Klein R, Klein BEK, Moss SE, Cruickshanks KJ. The Wisconsin Epidemiologic Study Of Diabetic Retinopathy. XVII. The 14-year incidence and progression of diabetic retinopathy and associated risk factors in type 1 diabetes. Ophthalmology. 1998;105(10):1801-5.

13. Kato S, Shiokawa A, Fukushima H, Numaga J, Kitano S, Hori S, et al. Glycemic control and lens transparency in patients with type 1 diabetes mellitus. Am J Ophthalmol. 2001;131(3):301-4.

14. Falck A, Laatikainen L. Diabetic cataract in children. Acta Ophthalmol Scand. 1998;76(2):238-40.

15. Di Benedetto AD, Aragona P, Romano G, Romeo G, Di Cesare E, Spinella R, et al. Age and metabolic control influence lens opacity in type 1, insulindependent diabetic patients. J Diabetes Complications. 1999;13(3):159-62. 